\title{
Geometric Crossovers for Real-code Representation
}

\author{
Yourim Yoon \\ School of Computer Science \& Engineering \\ Seoul National University \\ Sillim-dong, Gwanak-gu, Seoul, 151-744, Korea \\ yryoon@soar.snu.ac.kr \\ Alberto Moraglio \\ Department of Computer Science \\ University of Essex \\ Wivenhoe Park, Colchester, CO4 3SQ, UK \\ amoragn@essex.ac.uk
}

Categories and Subject Descriptors: G.0 [Mathematics of Computing]: General

General Terms: Theory

Keywords: Geometric crossover, real-code representation

\section{INTRODUCTION}

Geometric crossover [1] is a representation-independent operator defined over the distance of the search space. Informally, geometric crossover requires the offspring to lie between parents. So far we have focused on the study of geometric crossover for combinatorial spaces and have left to the intuition the case of continuous space. In this paper we start to study the geometric crossovers for continuous spaces. A natural starting point are Minkowski distances that are simple generalizations of the Euclidean distance. Geometric crossovers based on Minkowski distances have an inherent bias toward the center of the space. This bias could be potentially harmful for the search. We then study geometric crossovers for glued versions of these spaces for which the bias disappears.

\section{GEOMETRIC CROSSOVERS}

Geometric crossover depends on the metric of given space. Although Euclidean distance is ordinarily used for $\mathbb{R}^{n}$, there exist many other metrics for $\mathbb{R}^{n}$. We study geometric crossover for real vector space under more general and abstract metric, Minkowski distance. The metric $d_{p}(x, y)$ is defined as $\|x-y\|_{p}$ on $\mathbb{R}^{n}$, where $\|\cdot\|_{p}$ is $p$-norm. Using these metrics, the metric segment between two points $x$ and $y$ on $\mathbb{R}^{n}$ under the metric $d_{p}$ induced by $p$-norm is defined as follows:

$[x ; y]_{d_{p}}=\left\{z \in \mathbb{R}^{n}:\|x-z\|_{p}+\|z-y\|_{p}=\|x-y\|_{p}\right\}$. Figure 1 shows an example of metric segments for the cases that $p=1,2$ and $\infty$. Geometric crossover takes offspring that lie in the metric segments. We design new geometric crossovers using the metric segments induced by $p$-norms by choosing offspring that lie in the segments.

Although new crossovers are theoretically meaningful, if they cannot be implemented efficiently, they are useless. We

Copyright is held by the author/owner(s).

GECCO'07, July 7-11, 2007, London, England, United Kingdom.

ACM 978-1-59593-697-4/07/0007.

\author{
Yong-Hyuk Kim \\ Department of Computer Science \\ Kwangwoon University \\ Wolgye-dong, Nowon-gu, Seoul, 139-701, Korea \\ yhdfly@kw.ac.kr \\ Byung-Ro Moon \\ School of Computer Science \& Engineering \\ Seoul National University \\ Sillim-dong, Gwanak-gu, Seoul, 151-744, Korea \\ moon@soar.snu.ac.kr
}
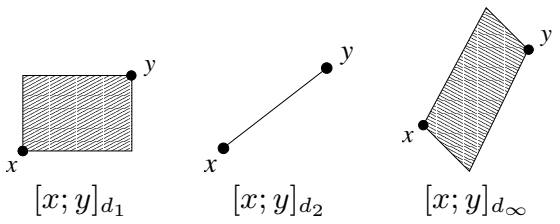

Figure 1: Metric segments induced by $p$-norms

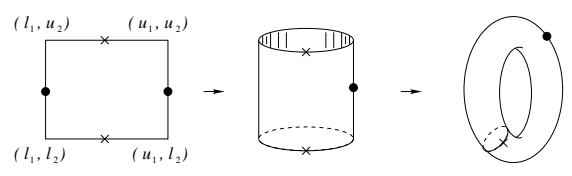

Figure 2: Glued space on $\mathbb{R}^{2}$

considered 1-norm, 2-norm and $\infty$-norm, and devised practical methods to implement geometric crossovers induced by the norms. However, it is omitted due to space constraints.

In general, solution space of real-coded problems has the range. Let the solution space $X$ be $\left\{x \in \mathbb{R}^{n}: l_{i} \leq x_{i}<\right.$ $u_{i}$ for each $\left.i\right\}$ where $l=\left(l_{1}, l_{2}, \ldots, l_{n}\right)$ is a lower bound and $u=\left(u_{1}, u_{2}, \ldots, u_{n}\right)$ is an upper bound. If we apply geometric crossover on this bounded domain, offspring have bias toward the center of the space. We propose a novel method to eliminate this bias. It is gluing the boundaries by identifying $u_{i}$ to $l_{i}$ for each $i$. Figure 2 shows this glued space for $\mathbb{R}^{2}$ case. Its explicit methodology and theoritical results are not presented here due to space limit.

In future work we want to implement the new crossovers presented in this paper and test them on problems for which we expect them to perform well from the study of their fitness landscapes.

\section{ACKNOWLEDGMENTS}

This work was supported by the Brain Korea 21 Project in 2007. The ICT at Seoul National University provided research facilities for this study.

\section{REFERENCES}

[1] Moraglio, A., And Poli, R. Topological interpretation of crossover. In Proceedings of the Genetic and Evolutionary Computation Conference (2004), pp. 1377-1388. 